\title{
Postscriptum: Skizze einer Systematisierung von Relationen zwischen Regimen des Engagements und Rechtfertigungsordnungen
}

In Abschn. 3.1.1 wurde die Regimetheorie als Bestandteil der Handlungstheorie der EC eingeführt. Dort wurde darauf hingewiesen, dass die Regimetheorie von Laurent Thévenot und Nicolas Auray im Anschluss an die Rechtfertigungstheorie in verschiedenen Beiträgen entwickelt und weiterentwickelt wurde (Thévenot 1990, 1994, 1995b; Auray 2007, 2011) $)^{1}$ Zugleich erfuhr die Regimetheorie in der Rezeption jedoch nicht dieselbe Reichweite wie die Rechtfertigungstheorie. Die empirische Analyse der verschiedenen Survey-Welten und Survey-Projekte hat jedoch regimebezogene Koordinationsherausforderungen in vielfältiger Weise ersichtlich gemacht. Zunächst hat sich gezeigt, dass Survey-Welten systematisch unterschiedlich auf verschiedene Regime Bezug nehmen. Deutlich wurde beispielsweise der starke Bezug der Informationswelt auf das Rechtfertigungsregime (vgl. Abschn. 5.4) oder die enge Verbindung zwischen der Beratungswelt und dem Regime des Vertrauten (vgl. Abschn. 5.2). Koordinationsprobleme und -herausforderungen zwischen Survey-Welten entpuppten sich in der Fallanalyse folglich wenig erstaunlich oft als Regimekonflikte (vgl. Abschn. 6.1.3, 6.1.4 und 6.2.4). Die Herausforderungen der Inter-Regime-Koordination wurden jedoch auch im Bereich des Befragtenmanagements ersichtlich. So war es zeitweise das Ziel in Fall (A), durch einen Wechsel in das Regime des Vertrauten validere Befragungsdaten $\mathrm{zu}$ erzielen (vgl. Abschn. 6.1.4). Deutlich wird dadurch insgesamt, dass die Koordination zwischen verschiedenen Regimen des Engagements, aber auch zwischen verschiedenen Regimen und Rechtfertigungsordnungen, $\mathrm{zu}$ spezifischen und systematischen

\footnotetext{
${ }^{1}$ Eine vollständige Auflistung der Texte, in welchen Thévenot die Regimetheorie weiterentwickelt hat, wäre schlicht zu umfangreich. Vgl. Abschn. 3.1.1 für eine Darstellung der Regimetheorie und deren Weiterentwicklungen.
} 
Koordinationsherausforderungen führt. Gleichzeitig fehlt es bis jetzt an einer systematischen Analyse und Ausarbeitung dieser koordinativen Übersetzungsschwierigkeiten. Das Ziel dieses Postscriptums liegt in einer Systematisierung der Übersetzungsschwierigkeiten einerseits zwischen verschiedenen Regimen des Engagements und andererseits zwischen verschiedenen Regimen des Engagements und Rechtfertigungsordnungen. Damit soll die Theoriebildung im Hinblick auf die Regime des Engagements angestoßen und damit auch die empirische Anwendbarkeit dieses Ansatzes gestärkt werden.

Nebst der Theorie der Rechtfertigungsordnungen ist die Theorie der Regime des Engagements der zweite Teil des für die EC grundlegenden Konzepts der doppelten Pluralität des Handelns, bzw. des Engagements (Diaz-Bone 2018, S. 387 ff.). Die Ausarbeitung der Regime des Engagements reflektiert jedoch nicht nur unterschiedliche durch Thévenot identifizierte Handlungslogiken, sondern setzt zugleich auch unterschiedliche sozialwissenschaftliche Handlungstheorien zueinander in Beziehung. So thematisiert die durkheimsche Soziologie gemäß Thévenot primär das Problem kollektiver Handlungslogiken. Diese Art der Handlungsorientierung wird in der Regimetheorie durch das Regime der Rechtfertigung thematisiert. Rational-Choice-Theorien fokussieren gemäß Thévenot auf das zielgerichtete Handeln, welches in der Regimetheorie durch das Regime des Plans aufgegriffen wird. Die phänomenologische Forschungstradition hingegen behandelt Prozesse der Vertrautheit und des unmittelbaren Erlebens, welche durch das Regime des Vertrauten in die Regimetheorie Eingang gefunden haben (Thévenot 2006a, S. 211). Die eigentliche Innovativität der Regime des Engagements liegt darin, die unterschiedlichen Perspektiven der verschiedenen Theorieansätze auf Handlung nicht als problematisch zu betrachten, sondern als Ergebnis tatsächlich existierender, unterschiedlicher Handlungslogiken. Durch die Verknüpfung der Regime des Engagements mit verschiedenen Theorietraditionen lässt sich argumentierten, dass der Erklärungsanspruch der Regimetheorie weniger in einer umfassenden Theoretisierung der verschiedenen Regime des Engagements, sondern im Aufzeigen der Übersetzungsprobleme zwischen den verschiedenen Regimen liegt. Der methodologische Fokus auf Übersetzungsprozesse zwischen verschiedenen Regimen des Engagements lässt sich an verschiedenen Arbeiten, welche mit der Regimetheorie arbeiten, ablesen. Beispiele hierfür stellen die Arbeiten von Eduardo Basto und Laura Centemeri (2014); von Centemeri (2015); von Laura Silva Castaneda (2012) und von Thévenot (2009b, S. 805 ff., 2011 c, S. 244 ff.) dar.

Einerseits konzipiert also auch Thévenot selbst Übersetzungen zwischen verschiedenen Regimen des Engagements als eigentlichen Fokus der Regimetheorie, andererseits mangelt es jedoch an einer Systematisierung dieser 
Übersetzungsverhältnisse. Dasselbe trifft auch auf das Verhältnis zwischen den verschiedenen Regimen des Engagements und Rechtfertigungsordnungen zu. Auch hier sieht Thévenot unterschiedliche Relationen zwischen den verschiedenen Handlungsregimen auf der einen Seite und den unterschiedlichen Rechtfertigungsordnungen auf der anderen Seite. Es fehlt jedoch auch hier an einer Systematisierung dieser Relationen (Thévenot 2011d, S. 270 f.).

Diese Situation ist für die empirische Forschung eine unbefriedigende Situation, da die Übersetzungshürde zwischen Regimen und Rechtfertigungsordnungen auch in den in dieser Arbeit untersuchten Fällen für Koordinationsprobleme sorgten. So stellt sich die in Fall (A) von Auftraggeber und Auftragnehmer identifizierte Sprachproblematik der Befragung bei genauerem Hinschauen als Regime-Konflikt heraus. Denn die Beantwortung von Survey-Fragen stellt generell den Anspruch, Vertrautes auf allgemeine Kategorien beziehen zu können. Damit wird aber unweigerlich der Anspruch an befragte Personen gestellt, eine Übersetzungsleistung primär vom Regime des Vertrauten in das Rechtfertigungsregime zu leisten. In Fall (B) zeigt sich die Übersetzungsproblematik zwischen Regimen am stärksten im durch die Survey-Firma kritisierten fehlenden Storytelling des durch den Auftraggeber formulierten Fragebogens. Das Einarbeiten des Storytellings soll gemäß der Survey-Firma dazu dienen, die verschiedenen Fragen sinnvoll in die Perspektive der Befragten zu integrieren. Folglich stellt sich auch hier das Problem einer Vermittlung von allgemeinen Kategorien an den vertrauten Kontext der Befragten.

Deutlich wird, dass sich die Perspektive der Regime des Engagements auf Übersetzungsprozesse auch in der vorliegenden Arbeit als gewinnbringender Ansatz herausgestellt hat. Gleichzeitig stellte sich jedoch die oben dargestellte, fehlende Systematisierung in der empirischen Anwendung als Hürde dar. Entsprechend soll die folgend vorgenommene Systematisierung von Übersetzungsverhältnissen von Regimen des Engagements einen Anstoß darstellen für eine weitere Ausarbeitung dieser Theorie, insbesondere auch für die empirische Erforschung von Koordinationsproblemen bei der Herstellung von Survey-Daten.

\section{1 Übersetzungsverhältnisse zwischen den verschiedenen Regimen des Engagements}

In den folgenden Absätzen wird eine Systematisierung der Relationen zwischen dem Regime der Rechtfertigung, des Plans und des Vertrauten vorgenommen. Obwohl Thévenot die Regime des Engagements als Gegenentwurf zur Habitus-Theorie von Pierre Bourdieu (Bourdieu 1993) betrachtet (Thévenot 2006b, S. 56, 2013, S. 173), 
wird in den folgenden Ausführungen auf Konzepte von Bourdieu zurückgegriffen. Das Ziel liegt dabei darin, durch den Bezug auf etablierte soziologische Konzepte die spezifische Stoßrichtung und den Beitrag der Theorie der Handlungsregime für die soziologische Handlungstheorie und das Verständnis von Sozialisierung aufzuzeigen. Zudem erlaubt die Übersetzung von bourdieuschen Elemente in die Theorie der Regime des Engagements eine Übernahme von bourdieuschen Problemstellungen, jedoch ohne deren marxistisches Erbe.

Ein von Thévenot vorgebrachtes Beispiel für die Übersetzung zwischen verschiedenen Regimen des Engagements stellt die Transformation von staatlichen Programmen der Sozialarbeit hin zu konkreten Massnahmen und weiter zu deren Vermittlung an hilfsbedürftige Personen dar (Thévenot 2011c, S. 245). Deutlich wird, dass dies eine Fähigkeit darstellt, welche von den involvierten Personen mehr oder weniger gut beherrscht werden kann. Man könnte folglich von einer Übersetzungskompetenz sprechen, welche es ermöglicht, diese Übersetzung vorzunehmen und dabei so weit wie möglich die verschiedenen Handlungslogiken miteinander in einen Kompromiss zu setzen. Diese Übersetzungskompetenz wird in den folgenden Absätzen durch den Verweis auf den Realitätssinn und den praktischen Sinn thematisiert. Mit Blick auf die drei hier thematisierten Regime des Engagements, dem Regime der Rechtfertigung, des Plans und des Vertrauten, existieren zwei Übersetzungshürden, welche durch kompetente Akteure bewältigt werden müssen.

Der erste Übergang besteht zwischen dem Regime der Rechtfertigung und dem des Plans. Bei dieser Übersetzung besteht die Notwendigkeit, individuelle Handlungspläne mit kollektiven, moralisch fundierten Logiken in eine Übereinstimmung zu bringen. Geklärt werden muss hier folglich, inwiefern individuelle Pläne in Einklang gebracht werden können mit den verschiedenen Rechtfertigungsordnungen. Aus der Perspektive des Rechtfertigungsregimes muss bei diesem Übergang geklärt werden, inwiefern rechtfertigungsbasierte Forderungen tatsächlich umsetzbar sind, d. h. in individuelle Pläne übersetzt werden können. In Anlehnung an Bourdieu lässt sich hier von einem Realitätssinn oder auch von einem sozialen Sinn sprechen. ${ }^{2}$ Das Konzept des Realitätssinns zielt bei Bour-

\footnotetext{
${ }^{2}$ Das Konzept des Realitätssinns und das später in diesen Ausführungen behandelte Konzept des praktischen Sinns scheinen bei Bourdieu nicht klar getrennt zu sein. So spricht er auch vom praktischen Sinn als dem „Sense of one's Place“ (Bourdieu 2004, S. 238), was in einer Trennung zwischen Realitätssinn und praktischem Sinn eher dem Realitätssinn entsprechen würde. Die Trennung zwischen dem Realitätssinn und dem praktischen Sinn bekommt jedoch durch den Bezug auf verschiedene Regime in den folgenden Ausführungen ihre genauere regimetheoretische Bedeutung.
} 
dieu auf die Abschätzung, bzw. die Umsetzungsmöglichkeit, von individuellen Plänen angesichts der objektiven Verhältnisse des sozialen Raums (Bourdieu 1979, S. 324). ${ }^{3}$ Aus der Perspektive der EC tritt jedoch an die Stelle des sozialen Raums eine Pluralität von strukturierenden Logiken (Diaz-Bone 2018, S. 380 ff.). Es ist aber aus der Perspektive der Regimetheorie exakt auch der von Bourdieu beschriebene Abgleich von kollektiven Koordinationslogiken mit individuellen Plänen, welcher der Übersetzung, bzw. der Koordination, zwischen dem Regime der Rechtfertigung und demjenigen des Plans entspricht.

Der zweite Übergang besteht zwischen dem Regime des Plans und demjenigen des Vertrauten. Geklärt werden muss bei diesem Übergang die Vermittlung zwischen individuellen Plänen und der persönlichen Annehmlichkeit. Einerseits stellt sich hier die Frage, wie das Versprechen der Annehmlichkeit des Regimes des Vertrauten durch das Verfolgen von individuellen Plänen erzielt werden kann. Andererseits muss bei diesem Übergang aus der Perspektive des Regimes des Plans geklärt werden, welche Konsequenzen das Verfolgen von zielgerichteten Handlungen für das Versprechen der Annehmlichkeit des Regimes des Vertrauten hat. Hierbei kann wiederum auf die bourdieusche Soziologie verwiesen werden, welche mit dem Konzept des praktischen Sinns auf die Leichtigkeit des Handelns in einer bekannten Umgebung verweist (Wacquant 2006, S. 43 ff.). Die von Bourdieu beschriebene Ökonomie der Logik des praktischen Sinns stellt aus der Perspektive der Regime des Engagements jedoch nicht primär das Resultat von geschlossenen Augen dar (Thévenot 2011e, S. 36). Es ist aus der Perspektive der EC folglich nicht lediglich der unbewusste Bezug auf Handlungsmuster, welches den praktischen Sinn ausmacht, sondern vielmehr die (aktive) Vermittlung der instrumentellen Logik des Plans an die Logik der Annehmlichkeit des Vertrauten. Aus der Perspektive der Regime des Engagements ist der praktische Sinn folglich nicht das Resultat von unbewussten, determinierenden sozialen Strukturen des sozialen Raums, sondern das Ergebnis eines pragmatischen Rückgriffs auf vorhandene Ressourcen, welche auf die unterschiedlichen Versprechen (bzw. „Güter") der verschiedenen Regime des Engagements ausgerichtet sind. Obwohl Marc Breviglieri das Konzept der „Handhabung“ primär auf das Regime des Vertrauten bezieht (Breviglieri 2004, S. 24). So wird dennoch deutlich, dass sich

\footnotetext{
${ }^{3}$ Der soziale Raum dient bei Bourdieu der Analyse der objektiven Klassenverhältnisse. Er beinhaltet drei Dimensionen: Die Gesamtheit des verfügbaren Kapitalvolumens, die Kapitalstruktur, d. h. der Anteil des kulturellen und ökonomischen Kapitals am Gesamtkapital und die Entwicklung des Verfügens über Kapital sowie der Kapitalstruktur über die Zeit (Bourdieu 1987, S. 195 ff.).
} 
die Handhabung auch auf zielgerichtetes Handeln beziehen kann. Entsprechend kann argumentiert werden, dass die Handhabung gerade aus einer Verknüpfung zwischen dem Regime des Vertrauten und demjenigen des Plans entsteht.

Die beiden Sinne für die Übersetzung zwischen verschiedenen Regimen des Engagements sind in Abb. 9.1 visuell dargestellt. Zudem sind die unterschiedlichen Übersetzungsverhältnisse abgebildet, welche in den folgenden Absätzen diskutiert werden.

In den folgenden Absätzen werden nun die verschiedenen Übersetzungsverhältnisse zwischen den Regimen des Engagements systematisch dargestellt.

Bereits beim Konzept der Forminvestition kann eine Interdependenz zwischen Regimen identifiziert werden (Thévenot 1984). Das Ziel des Konzepts der Forminvestition ist es aufzuzeigen, wie Handlungslogiken auf Dauer gestellt werden. Folgt man dem Beispiel Thévenots hierfür, dem Taylorismus, so lässt sich behaupten, dass hier eine Handlungslogik, welche aus der industriellen Rechtfertigungsordnung abgeleitet wurde, in der täglichen Arbeit auf Dauer gestellt werden soll (Thévenot 1984, S. 6 ff.). Handlungslogiken des Rechtfertigungsregimes sollen folglich ins planende Handeln übersetzt werden. Dies geschieht durch die Einlagerung von Dispositiven in das planende Handeln. Es sind also (objektbasierte) Dispositive, welche die „Rechtfertigbarkeit“ des planenden Handelns gewährleisten sollen. ${ }^{4}$ Es kann folglich argumentiert werden, dass das tägliche Handeln in Organisationen nicht per se auf Rechtfertigungsordnungen fußt, sondern dass es Dispositive als „Stützen der Handlung“ sind, welche Rechtfertigungsordnungen an das planende Handeln vermitteln.

Als Moralisierung kann der Bezug von Handlungen des Regimes des Plans auf Rechtfertigungsordnungen bezeichnet werden. Vormals „unproblematische“, lediglich instrumentelle Handlungen werden durch den Bezug auf Rechtfertigungsordnungen einem rechtfertigungsbasierten Test unterzogen. Das lediglich zweckbasierte Handeln wird folglich unterbrochen und einer rechtfertigungsbasierten Neubewertung unterzogen. Beispiele hierfür stellen der Fleischkonsum oder der Mobilitätsgebrauch dar. Waren beide Tätigkeiten lange Zeit lediglich einer individuellen Planlogik unterworfen, ${ }^{5}$ werden zunehmend moralische Kategorien an diese Tätigkeiten herangetragen, insbesondere auf Basis der grünen Rechtfertigungsordnung.

\footnotetext{
${ }^{4}$ Boltanski und Thévenot gehen davon aus, dass Personen im täglichen Leben ständig zwischen Reflexion und Handeln wechseln (2007, S. 478).

${ }^{5}$ Hier lässt sich natürlich argumentieren, dass allenfalls ein Kompromiss mit dem Regime des Vertrauten existiert.
} 


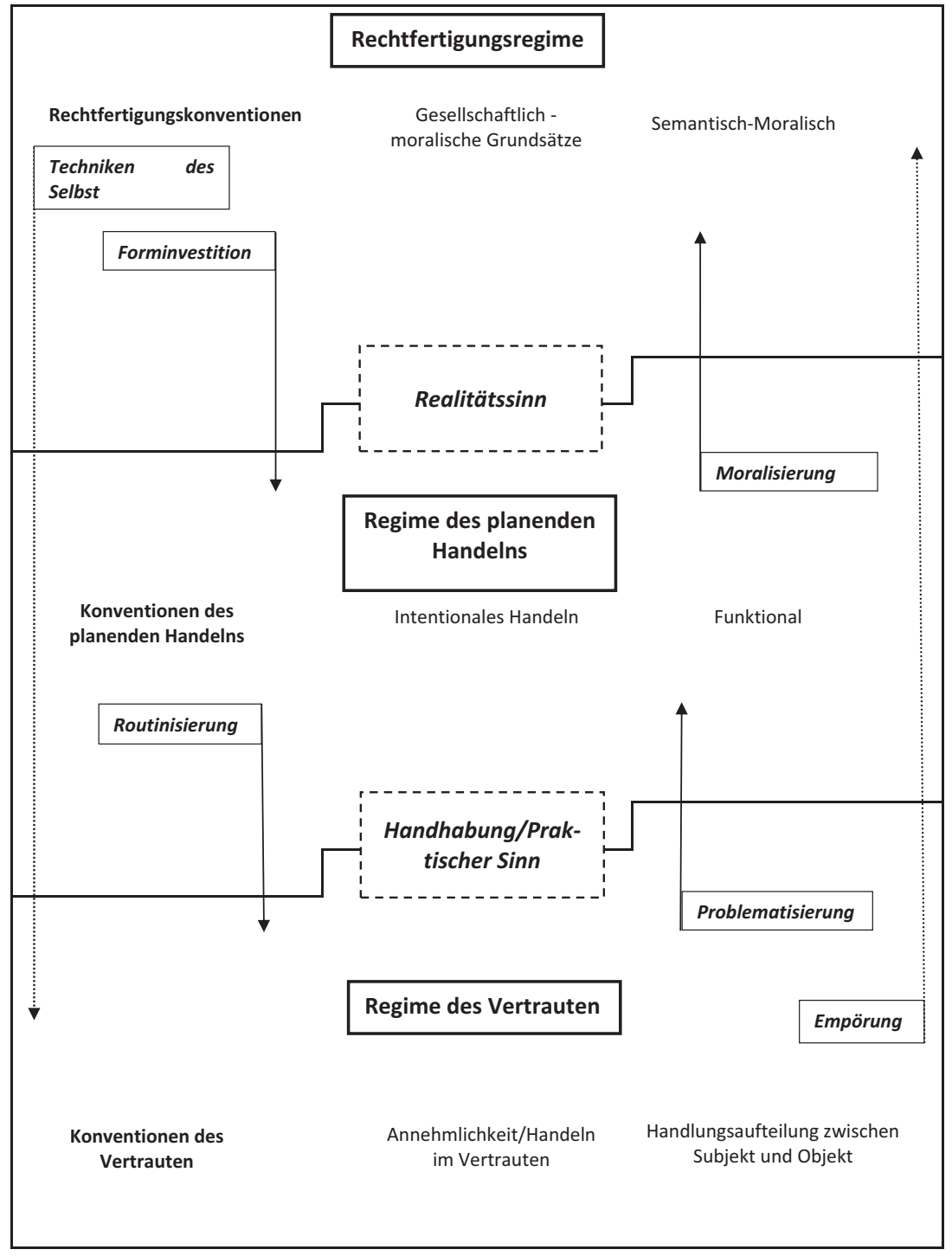

Abb. 9.1 Übersetzungsverhältnisse zwischen verschiedenen Regimen des Engagements 
Analog kann nach Interdependenzen des Regimes des Vertrauten gefragt werden. Das Vertrautwerden, das Erreichen einer Annehmlichkeit bedingt einer Urbarmachung. Das Verhältnis zur Welt ist zunächst ein aktives, in welchem Gegenstände, aber auch Personen, erst durch den wiederholten (aktiven) Umgang ihre überraschenden Eigenheiten und Widerständigkeiten verlieren. Die Welt wird folglich zunächst durch aktive Handlungen erschlossen. Das Suchen des Plans nach Funktionsweisen und Verwendbarkeiten wird erst nach und nach abgelöst durch einen Umgang mit Dingen und Personen im Vertrauten, in welchem das Handeln infolge der Koevolution zwischen Objekten und Akteur die Notwendigkeit der Reflexion verliert. Diese Bewegung vom Regime des planenden Handelns hin zum Regime des Vertrauten kann als Routinisierung bezeichnet werden. ${ }^{6}$

Eine Problematisierung beginnt dann, wenn das Vertraute nicht mehr in den gewohnten Bahnen abläuft. Dies kann gut mit dem Prozess der Einführung von neuer Software illustriert werden. Eigentlich routinehafte Handlungen müssen hier erst wieder mühsam eingeübt werden, gewohnte Funktionsweisen und Abkürzungen funktionieren nicht mehr wie gewohnt. Es findet folglich eine Problematisierung von gewohnten, routinehaften Handlungen statt. Liefen viele Handlungen in der alten Software intuitiv ab, müssen diese jetzt unter Beachtung der Funktionsweise der neuen Software Schritt für Schritt ,aktiv“ ausgeführt werden. Erst nach einer aufwendigen Einarbeitungszeit funktioniert auch der Umgang mit der neuen Software wieder mühelos und diese wird so wieder zur ,zweiten Heimat".

Gemäß Thévenot ist es ein Charakteristikum der neuen sozialen Bewegungen, Bedürfnisse und Problematiken des Regimes des Vertrauten politisch und öffentlich zu artikulieren (Thévenot 2006a, S. 220). Adressiert werden hier folglich nicht handlungspraktische Probleme, sondern vielmehr beispielsweise Themen der Identität, der gefühlten Sicherheit etc., welche dem Regime des Vertrauten entspringen. Diese direkte Übersetzung von Anliegen vom Regime des Vertrauten in das Rechtfertigungsregime kann als Empörung bezeichnet werden. Denn de facto findet hierbei kein Bezug auf die handlungspraktischen Probleme des regulären

\footnotetext{
${ }^{6}$ Dieses Abstützen auf Routinen kann dann auch problematische Folgeeffekte mit sich ziehen, wenn diese im Notfall nicht mehr auf die offiziell kodifizierten Formen rückbezogen werden können. Vgl. hierfür die Studie von Brian Wynne zur Entwicklung von Routinen jenseits von offiziellen Vorgaben und der daraus folgenden Konsequenz für technische Notfälle (1988).
} 
Politikbetriebs statt. Entsprechend schwierig ist es oft, eine Integration der Anliegen der neuen sozialen Bewegungen in den regulären Politikbetrieb zu gewährleisten.

Eine der Kernthemen von Michel Foucault stellt die Thematisierung von sogenannten Techniken des Selbst dar. Gemeint sind damit Prozesse der Formung der eigenen Persönlichkeit durch den Bezug auf Diskursformationen (Foucault 1977, 1993). Foucault umschreibt Technologien des Selbst folgendermaßen:

Technologien des Selbst, die es dem Einzelnen ermöglichen, aus eigener Kraft oder mit Hilfe anderer eine Reihe von Operationen an seinem Körper oder seiner Seele, seinem Denken, seinem Verhalten und seiner Existenzweise vorzunehmen, mit dem Ziel, sich so zu verändern, daß er einen gewissen Zustand des Glücks, der Reinheit, der Weisheit, der Vollkommenheit oder der Unsterblichkeit erlangt (Foucault 1993, S. 26).

Deutlich wird hier, dass Foucault mit dem Konzept von Technologien des Selbst die Arbeit an sich selbst und den (vertrauten) Praktiken meint. Aus der Perspektive der Regime des Engagements kann dies als Bezug von Rechtfertigungsordnungen auf das Vertraute verstanden werden. ${ }^{7}$ Deutlich wird aus der Perspektive der Regime des Engagements, dass sich diese Techniken nicht notwendigerweise auf das Subjekt reduzieren müssen, sondern auch auf Stützobjekte des Vertrauten beziehen können. Denn wie Thévenot im Hinblick auf das Regime des Vertrauten deutlich machte, ist dieses Regime gerade durch eine Distribution von Handlung auf Akteure und Objekte gekennzeichnet (Thévenot 2011d, S. 266 f.).

\subsection{Relationen zwischen Rechtfertigungsordnungen und Regimen des Engagements}

Handlungsregime sind gemäß Thévenot eigenständige Handlungslogiken. Auch die Sozialisierung in den einzelnen Regimen ist regimespezifisch (Thévenot 2006a, S. 237). Trotz dieser Eigenständigkeit der Handlungsregime untereinander bestehen jedoch unterschiedlich starke Affinitäten zwischen den verschiedenen Handlungsregimen und Rechtfertigungsordnungen (Thévenot 2011d, S. 270). Thévenot beschreibt jedoch nur einzelne Relationen zwischen Rechtfertigungs-

\footnotetext{
${ }^{7}$ Thévenot sieht in den Ausführungen von Foucault eine Koordination mit sich selbst (Thévenot 2013, S. 164).
} 
ordnungen und Handlungsregimen. Das Ziel der folgenden Ausführungen besteht darin, einen ersten Schritt hin zu einer Systematisierung von Relationen zwischen verschiedenen Rechtfertigungsordnungen und Handlungsregimen $\mathrm{zu}$ tun. Es werden analytisch auf Basis der unterschiedlichen Handlungslogiken von Rechtfertigungsordnungen und Handlungsregimen verschiedene Relationen herausgearbeitet. ${ }^{8}$

Die marktweltliche Rechtfertigungsordnung ist durch ein neutrales Verhältnis zum Regime des Plans gekennzeichnet. ${ }^{9}$ Denn einerseits ist diese Rechtfertigungsordnung auf eine Systematik des Handelns angewiesen, andererseits bauen Märkte auf einer gewissen Neugier von Kunden auf, wie noch aufgezeigt werden soll. Hingegen existiert ein problematisches Passungsverhältnis zum Regime des Vertrauten. Denn trotz des Versprechens der Marktwelt einer Befriedigung individueller Wünsche, existiert auch in der Marktwelt eine Standardisierung, welche sich in einer Objektivität der gehandelten Güter zeigt (Boltanski und Thévenot 2007, S. 266 f.). Entsprechend muss auch in der Marktwelt ein Kompromiss gefunden werden zwischen der radikalen Individualität des Regimes des Vertrauten und der standardisierten „Individualität“ der Marktwelt. Dieser Übergang kann jedoch eine Quelle von Koordinationsproblemen sein, wenn die Standardisierung des Marktes das Gut der Annehmlichkeit des Regimes des Vertrauten bedroht. Im Gegensatz dazu existiert ein Wahlverhältnis zwischen der marktweltlichen Rechtfertigungsordnung und dem Regime der Exploration. Das Kompromissobjekt zwischen beiden Handlungslogiken stellt das „Interesse“ dar. Das Interesse ist dabei dasjenige Konzept, welche die Menschen in der Marktwelt zusammenbringt (Boltanski und Thévenot 2007, S. 274). Die Neugierde als treibender Aspekt des explorativen Regimes wird in modernen Märkten immer mehr dazu verwendet, um ein Interesse bei potenziellen Kunden zu wecken (Thévenot 2014b, S. 14). Dieser Umstand ist einer der kritisierten Aspekte, wenn auf das grundsätzlich unendliche Wachstumspotenzial des Kapitalismus hingewiesen wird. Eine Voraussetzung dafür ist jedoch die

\footnotetext{
${ }^{8}$ Die Systematisierung basiert folglich nicht auf empirischer Forschung, sondern stellt eine rein analytische Einteilung dar.

${ }^{9}$ Die Relation zwischen dem Rechtfertigungsregime und dem Regime des Plans ist generell durch ein ambivalentes Verhältnis gekennzeichnet. Einerseits sind Rechtfertigungsordnungen auf das Regime des Plans angewiesen, um eine konkrete Umsetzung zu erfahren. Andererseits stellt die Zweckrationalität des Regimes des Plans auch eine Gefahr für das Rechtfertigungsregime dar, da das Beachten von Rechtfertigungsordnungen beim zielgerichteten Handeln untergehen kann.
} 
Bereitschaft der Kunden, sich auf Neues einzulassen und Neues entdecken zu wollen, was die Basis darstellt für neuartige Wünsche. Und genau diese Voraussetzung adressiert die Handlungslogik des Regimes der Exploration. ${ }^{10}$ Zudem ist der Bezug der marktweltlichen Rechtfertigungsordnung auf das Regime der Exploration auch die Bedingung für innovationsgetriebene Märkte. Denn hier müssen konstant Neuigkeiten entwickelt werden, was aber eine Neugier und die Bereitschaft für neue Ansätze voraussetzt.

Die industrielle Rechtfertigungsordnung ist wie keine andere Rechtfertigungsordnung mit der Rationalität des Regimes des Plans kompatibel. Entsprechend existiert ein starkes Wahlverhältnis zwischen der auf Methoden, Standards und Normierungen aufbauenden industriellen Rechtfertigungsordnung (Boltanski und Thévenot 2007, S. 276 ff.) und der planenden und voraussichtigen Handlungslogik des Regimes des Plans (Thévenot 2011d, S. 267 f.). Hingegen steht diese Rechtfertigungsordnung in einem problematischen Verhältnis sowohl zum Regime des Vertrauens als auch zu demjenigen der Exploration. ${ }^{11}$ Denn einerseits liegt der Sinn von Standards etc. gerade darin, das Individuelle zu verhindern. Andererseits sollen damit auch spontane Einführungen von neuen Handlungsprinzipien verhindert werden. Sowohl das individuell Vertraute, wie auch das individuell Explorative müssen aus der Perspektive der industriellen Rechtfertigungsordnung durch normierte Prozesse beherrschbar gemacht werden. ${ }^{12}$ Die Stoßrichtung der industriellen Rechtfertigungsordnung zielt folglich gerade auf eine Kontrolle der Handlungslogiken des Regimes des Vertrauten sowie der Exploration.

\footnotetext{
${ }^{10}$ Wohl nicht zufällig identifizierte Nicolas Auray das Regime der Exploration anhand von Informationstechnologien (Auray 2011). Diese relativ neue Industrie hat wie kaum ein anderer Wirtschaftszweig vor ihr neue Bedürfnisse und Wünsche von Kunden erschaffen und ist damit eng an die Bereitschaft der Exploration von Neuem gebunden.

${ }^{11}$ Die Unterdrückung des Vertrauten durch die industrielle Rechtfertigungsordnung wurde von Thévenot angemerkt (Thévenot 2011d, S. 270).

${ }^{12}$ Entsprechend kann in einem Vergleich mit der Marktwelt eine grundlegend unterschiedliche Perspektive auf die regimetheoretische Basis von Märkten festgestellt werden. Stellt für die marktweltliche Rechtfertigungsordnung das Regime der Exploration eine Quelle für das Entstehen von neuen Märkten dar, ist dieses Regime für die industrielle Rechtfertigungsordnung eher eine Bedrohung, da das Erschließen neuer Märkte für die industrielle Rechtfertigungsordnung einen größeren Aufwand bedeutet als für die marktweltliche Rechtfertigungsordnung und dadurch größere Risiken bestehen. Entsprechend findet eine stärkere Orientierung an existierenden Märkten durch die industrielle Rechtfertigungsordnung statt.
} 
Die häusliche Rechtfertigungsordnung basiert maßgeblich auf Traditionen (Boltanski und Thévenot 2007, S. 228 ff.). Entsprechend lässt sich hier kein spezifisches Verhältnis zum Regime des Plans identifizieren. Denn solange Traditionen Eingang finden in das planende Handeln, besteht kein Konflikt zur häuslichen Rechtfertigungsordnung. Hingegen existiert in der häuslichen Rechtfertigungsordnung die Vorstellung, dass Tätigkeiten eine starke Verankerung haben sollen, bzw. eine Natürlichkeit haben müssen, welche auf Gewohnheiten basiert (Boltanski und Thévenot 2007, S. 232). ${ }^{13}$ Entsprechend ist beispielsweise ein Beruf in dieser Welt nicht nur ein Mittel für den Broterwerb, sondern primär auch eine Berufung. Folglich findet durch diese Rechtfertigungsordnung ein starker Zugriff auf das Regime des Vertrauten statt. Denn wie bereits im Konzept der „Berufung“ enthalten, lässt sich hier nicht zwischen der beruflichen und der privaten Rolle unterscheiden. Die häusliche Rechtfertigungsordnung basiert so stark auf dem Konzept der „Verbundenheit“, was genau die Kopplung zwischen dieser Rechtfertigungsordnung und dem Regime des Vertrauten bezeichnet. Im Gegensatz dazu steht die häusliche Rechtfertigungsordnung in einem problematischen Verhältnis zum Regime der Exploration. Denn während die häusliche Rechtfertigungsordnung auf der Bewahrung von Traditionen aufbaut, sucht das Regime der Exploration nach dem Neuen (Auray 2011), welches eine Gefahr für Traditionen darstellt.

Bei der staatsbürgerlichen Rechtfertigungsordnung lässt sich gegenüber dem Regime des planenden Handelns eine neutrale Perspektive identifizieren. Einerseits kann das planende Handeln in einem Widerspruch zum kollektiven Interesse der staatsbürgerlichen Rechtfertigungsordnung stehen, andererseits ist ein (kollektives) strukturiertes Vorgehen für das Erreichen des kollektiven Interesses unumgänglich. Gegenüber dem Regime des Vertrauten besteht ein ambivalentes

\footnotetext{
${ }^{13}$ Vgl. hierfür auch die Ausführungen von Boisard zur traditionellen (häuslichen) Herstellungsart von Käse: „The appropriate time for coagulation varies from one day to the next, and the true skill of a cheesemaker is to judge by sight or touch the right moment to start the molding. According to one experienced cheesemaker, it is the person in charge of cheese making who personally makes the decision to begin molding [...]" (Boisard 1991, S. 184). Deutlich wird hier die zwischen Objekt und Subjekt distribuierte Handlungslogik des Regimes des Vertrauten (Thévenot 2011d, S. 266 f.), welche infolge eines Rückgriffs auf Erfahrung die spezifische Qualität des traditionellen Käses erst ermöglicht. „Erfahrung“ kann dabei als Ko-Konstruktion zwischen den Werten und Wissenskonzepten der häuslichen Rechtfertigungsordnung und einer spezifischen Sozialisierung im Vertrauten verstanden werden.
} 
Verhältnis. Denn hier besteht einerseits ein Konflikt zwischen dem kollektiven Interesse der staatsbürgerlichen Welt, welches über individuelle Verhältnisse hinweg Geltung erhalten muss und der auf Individualität abzielenden Handlungslogik des Regimes des Vertrauten (Boltanski und Thévenot 2007, S. 254) ${ }^{14}$ Denn die Orientierung am Kollektiv bedingt gerade der Zurücknahme von subjektiven Befindlichkeiten. ${ }^{15}$ Andererseits schützt die staatsbürgerliche Rechtfertigungsordnung durch Gesetze auch das Vertraute. Die grundlegende Institution hierfür stellen beispielsweise Bürgerrechte und Datenschutzgesetze dar. Denn dadurch werden gesetzliche Vorkehrungen für den Schutz der Privatsphäre getroffen. Gegenüber dem Regime der Exploration besteht ein kritisches Verhältnis. Denn die (individuelle) Neugier des Regimes der Exploration bedroht die Orientierung am Kollektiv der staatsbürgerlichen Rechtfertigungsordnung.

Die Rechtfertigungsordnung der Meinung ist eher indifferent gegenüber den verschiedenen Handlungsregimen. Dies betrifft insbesondere ihr Verhältnis zum Regime des Plans. Problematisch kann jedoch das Verhältnis zum Regime des Vertrauten sein. Denn einerseits ist für die öffentliche Kommunikation des Vertrauten eine Abstraktion notwendig. Die öffentliche Kommunikation verfälscht die Eigenheiten des Regimes des Vertrauten also in hohem Maße. Zudem nimmt die öffentliche Meinung kaum Rücksicht auf individuelle Befindlichkeiten (Boltanski und Thévenot 2007, S. 249). Dies zeigt sich im „öffentlichen Verriss“ von Personen, bzw. der ungefilterten Kritik an öffentlichen Personen, welche keine Rücksicht auf das persönliche Befinden der betroffenen Personen nimmt. Gegenüber dem Regime der Exploration ist die Rechtfertigungsordnung der Meinung indifferent.

Bei der Rechtfertigungsordnung der Inspiration lassen sich unterschiedliche Relationen zu den verschiedenen Handlungsregimen identifizieren. Zunächst lässt sich ein Konflikt zum Regime des Plans feststellen. Denn Inspiration passiert gerade dort, wo aus dem zielgerichteten Handeln ausgebrochen wird (Boltanski

\footnotetext{
${ }^{14}$ Übersetzungen zwischen der staatsbürgerlichen Rechtfertigungsordnung und dem Regime des Vertrauten werden gemäß Thévenot durch neue soziale Bewegungen angestrebt und erzielt (Thévenot 2006a, S. 220).

${ }^{15}$ Diese Eigenschaft der staatbürgerlichen Rechtfertigungsordnung zeigt sich bereits in den ersten beiden Sätzen der Ausführungen von Boltanski und Thévenot zur staatsbürgerlichen Rechtfertigungsordnung: „Die staatsbürgerliche Welt unterscheidet sich dadurch von den anderen Welten, dass solchen Wesen höchste Bedeutung zukommt, die keine Personen sind. In dieser Welt gelangen nämlich nicht Menschen zu wahrer Größe, sondern Kollektivpersonen [...]“ (Boltanski und Thévenot 2007, S. 254). Die Vertretung dieses Kollektivs durch Politiker bedingt folglich der Zurücknahme alles Individuellen.
} 
und Thévenot 2007, S. 222). Handeln im Regime des Plans steht deswegen gerade in einem Gegensatz zum Ziel der Inspiration. Auch das Gut der Annehmlichkeit des Regimes des Vertrauten lässt sich kaum mit der Rechtfertigung der Inspiration in Einklang bringen. Denn gemäß Boltanski und Thévenot ist es für wahre Inspiration notwendig, das „Vertraute unvertraut“ (Boltanski und Thévenot 2007, S. 223) zu machen. Die gewohnten Bahnen der Annehmlichkeit müssen deshalb hinter sich gelassen werden. Inspiration bedingt grundlegend, gewohnte Wege zu verlassen, wodurch ein positives Verhältnis zum Regime der Exploration besteht. Denn Inspiration kann nur denjenigen treffen, der auch offen ist für neue Erfahrungen (Boltanski und Thévenot 2007, S. 225).

Bei der grünen Rechtfertigungsordnung lassen sich keine grundlegend problematischen Beziehungen zu den verschiedenen Regimen identifizieren, jedoch auch kaum ausgesprochene Wahlverwandtschaften. Sie verhält sich gegenüber dem Regime des Plans indifferent. Denn auch hier gilt - wie bei sämtlichen Rechtfertigungsordnungen mit einem neutralen Verhältnis zu diesem Regime dass das Regime des Plans nicht problematisch ist, sofern darin die spezifischen Handlungslogiken der betreffenden Rechtfertigungsordnung beachtet werden. Im Hinblick auf das Regime des Vertrauten kann eine Ambivalenz festgestellt werden. Denn einerseits fordert die grüne Rechtfertigungsordnung eine Abkehr von der stark auf das Lokale begrenzten Perspektive des Regimes des Vertrauten. Folglich lässt sich ein Reichweitenkonflikt zwischen beiden Handlungslogiken identifizieren. Andererseits zeichnet sich das Regime des Vertrauten gerade durch die Distribuiertheit der Koordination zwischen Akteur und Objekten aus (Thévenot 2011d). Entsprechend besteht im Bezug auf dieses Handlungsregime auch eine Ressource für die rechtfertigungsbasierten Forderungen der grünen Rechtfertigungsordnung, da die dezentrale Perspektive auf Menschen hier vorbereitet ist. Dieser Effekt kann bei Forderungen der Tierrechtsbewegung festgestellt werden, wenn beispielsweise die Kategorisierung von Nutz- und Haustieren problematisiert wird. Hier wird oft die vertraute Nähe zu Haustieren aufgegriffen und eine Verallgemeinerung dieses vertrauten Status eingefordert. Im Verhältnis zum Regime der Exploration lässt sich keine spezifische Beziehung identifizieren.

Bei der netzwerkbasierten Rechtfertigungsordnung besteht ein positives Verhältnis zum Regime des Plans. Denn Projekte werden initiiert, um konkrete Handlungsprobleme zu lösen. Hingegen existiert ein gewisses Konfliktverhältnis zum Regime des Vertrauten. Das zentrale Prinzip der netzwerkbasierten Rechtfertigungsordnung besteht in der gemeinsamen Arbeit an einem Projekt durch verschiedene und stets aufs Neue zusammengesetzten Personen (Boltanski und Chiapello 2006, S. 154 ff.). Dies kann jedoch zu einem Konflikt mit der 
persönlichen Annehmlichkeit führen, da eine Anpassung an Regeln und Konventionen der kollektiven Projektarbeit notwendig wird. Zudem steht die netzwerkbasierte Rechtfertigungsordnung in einem Konflikt mit Arbeitsroutinen und einer spezifischen Handhabung der Arbeitstätigkeiten, da diese immer wieder neu auf Projekte ausgerichtet werden müssen (Thévenot 2011d, S. 271). Nicht zuletzt erfordert die Projektkultur oft eine hohe Mobilität, welche in einem Konflikt mit der Verankerung der Person in ihrem lokalen Umfeld steht (Thévenot 2011d, S. 267 f.). ${ }^{16}$ Diesen Umstand könnte man mit dem Begriff der „Entwurzelung“ beschreiben. Hingegen lässt sich ein Passungsverhältnis zwischen der Rechtfertigungsordnung der Meinung und dem Regime der Exploration identifizieren. Denn diese Rechtfertigungsordnung basiert gerade auf dem ständigen Austausch zwischen Personen mit unterschiedlichen Hintergründen und Fachgebieten (Boltanski und Chiapello 2006, S. 162 f.). Entsprechend ist eine gewisse Neugier unabdingbar, damit die verschiedenen Perspektiven in das Projekt integriert werden können und so von der Projektarbeit auch gelernt werden kann.

In Tab. 9.1 werden die verschiedenen Relationen zwischen Handlungsregimen und Rechtfertigungsordnungen tabellarisch dargestellt. Problematische Relationen sind dabei durch ,--“ dargestellt, neutrale durch ,0“, positive Relationen durch ,,+“ und ausgesprochene Wahlverhältnisse zwischen Rechtfertigungsordnungen und Regimen des Engagements durch ,,++“:17

\section{$9.3 \quad$ Fazit}

Wie zu Beginn dieses Kapitels argumentiert, wurden in den vorangehenden Absätzen Denkanstöße entwickelt für eine Systematisierung der Übersetzungsperspektive der Regimetheorie von Thévenot. Einerseits wurden dabei Übersetzungsverhältnisse zwischen Regimen des Engagements thematisiert. Das Ziel lag hier darin, eine Typisierung von Übersetzungsverhältnissen vorzunehmen und dadurch einen ersten Schritt zu einer Systematik der Übersetzung zwischen Regimen des Engagements zu machen. Daran anschließend wurden unterschiedliche Übersetzungsverhältnisse zwischen Rechtfertigungsordnungen und Regimen des Engagements identifiziert. Obwohl Regime des Engagements gemäß Thévenot

\footnotetext{
${ }^{16}$ Vgl. hierfür auch die Ausführungen von Richard Sennett zum „flexiblen Menschen“ als Akteursform des modernen Kapitalismus (2000).

${ }^{17}$ „Rechtfertigungsordnung“ ist in der folgenden Tabelle mit „RO“ abgekürzt.
} 


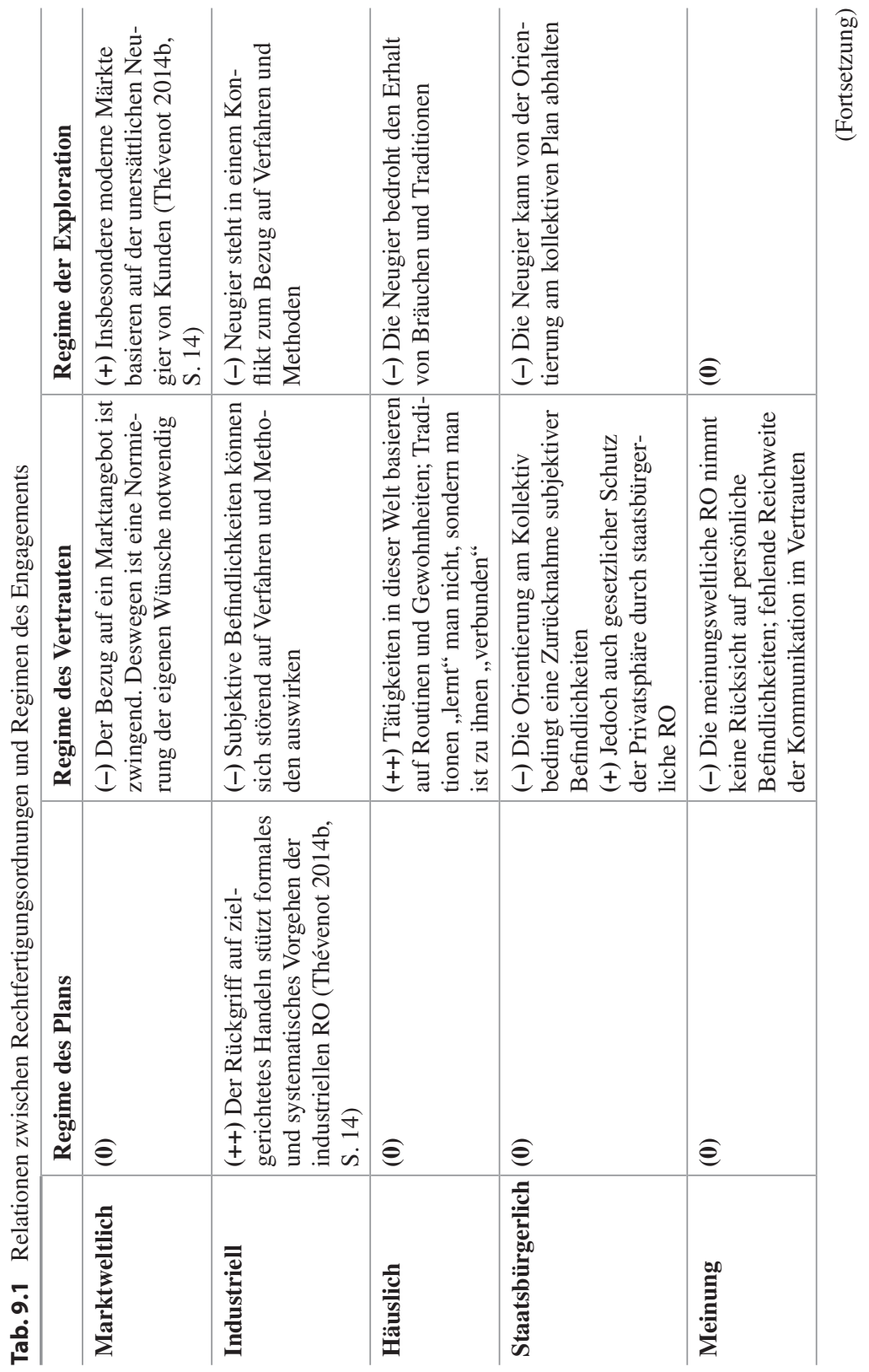




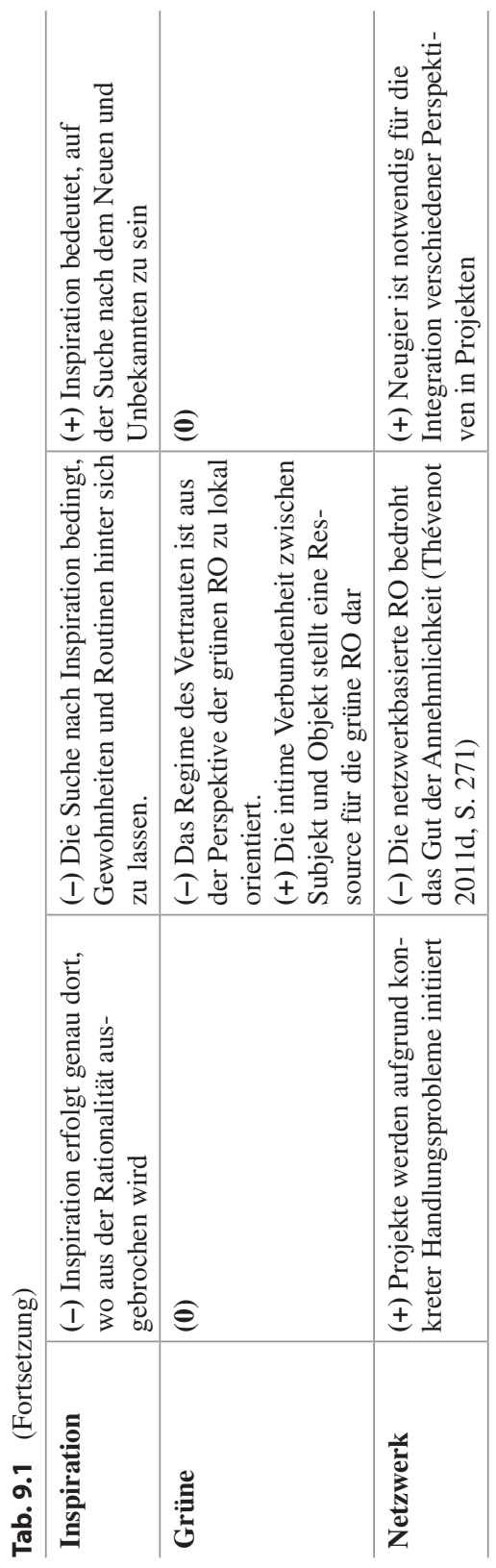


unterschiedliche Handlungslogiken darstellen, so wird dennoch deutlich, dass zwischen ihnen unterschiedliche Affinitäten bestehen. So konnten sowohl negative, neutrale als auch positive Relationen zwischen Rechtfertigungsordnungen und Regimen des Engagements identifiziert werden und darüber hinaus auch ausgesprochene Wahlverhältnisse.

Die vorgenommene Systematisierung der Übersetzungsverhältnisse zwischen verschiedenen Regimen des Engagements einerseits und Regimen des Engagements und Rechtfertigungsordnungen andererseits, ermöglicht eine engere Verzahnung des Konzepts der doppelten Pluralität in der EC (Diaz-Bone 2018, S. 387 ff.). Möglich wird damit eine systematische Perspektive auf Übersetzungsprozesse zwischen beiden Dimensionen der durch die EC angenommenen Pluralität von Handlungslogiken. Damit wird eine systematisierte Konfliktperspektive auf die Relationen zwischen Rechtfertigungsordnungen und Regimen des Engagements möglich, welche durch Thévenot bereits im Hinblick auf das Verhältnis von unterschiedlichen Rechtfertigungsordnungen eingeführt wurde (Thévenot 2002, S. 184). Verständlich werden dadurch Konflikte und Widerstände bei der Anwendung von Rechtfertigungsordnungen. Denn auf der Basis der Systematisierung der Übersetzungsrelationen zwischen Rechtfertigungsordnungen und Regimen des Engagements kann die Kollektivität solcher vermeintlich individueller Prozesse aufgezeigt werden.

In diesem Kapitel wurde eine Systematisierung der Übersetzungsverhältnisse zwischen verschiedenen Regimen des Engagements entworfen. Diese Systematisierung schließt jedoch auch direkt an die Rechtfertigungstheorie von Boltanski und Thévenot an. Denn bereits in „Über die Rechtfertigung“ wird der Wechsel zwischen dem alltäglichen Handeln und dem reflexiven Bezug des Handelns auf Rechtfertigungsordnungen erwähnt (Boltanski und Thévenot 2007, S. 478). Dieser Wechsel kann auf Basis der Regimetheorie von Thévenot als Regimewechsel beschrieben werden. Zentral ist dabei der Wechsel von einem funktionalen Regime zu einem semantischen Handlungsregime. Eine für die Regimetheorie spannende Frage stellt sich folglich, wann eine Semantisierung, bzw. eine Entsemantisierung stattfindet und wodurch diese ausgelöst wird. Zusätzlich steht eine Systematisierung der Übersetzungsverhältnisse zum Regime der Exploration noch aus. 
Open Access Dieses Kapitel wird unter der Creative Commons Namensnennung 4.0 International Lizenz (http://creativecommons.org/licenses/by/4.0/deed.de) veröffentlicht, welche die Nutzung, Vervielfältigung, Bearbeitung, Verbreitung und Wiedergabe in jeglichem Medium und Format erlaubt, sofern Sie den/die ursprünglichen Autor(en) und die Quelle ordnungsgemäß nennen, einen Link zur Creative Commons Lizenz beifügen und angeben, ob Änderungen vorgenommen wurden.

Die in diesem Kapitel enthaltenen Bilder und sonstiges Drittmaterial unterliegen ebenfalls der genannten Creative Commons Lizenz, sofern sich aus der Abbildungslegende nichts anderes ergibt. Sofern das betreffende Material nicht unter der genannten Creative Commons Lizenz steht und die betreffende Handlung nicht nach gesetzlichen Vorschriften erlaubt ist, ist für die oben aufgeführten Weiterverwendungen des Materials die Einwilligung des jeweiligen Rechteinhabers einzuholen. 